\title{
The Level of Certain Interleukins and Tissue Factor in Conditions of Intestinal Ischemia and Reperfusion in Rats
}

\author{
Zoya A. Artamonova, PhD; Evgeny V. Namokonov, PhD, ScD; Marina A. Darenskaya, $\mathrm{PhD}, \mathrm{ScD}$; \\ Natalya V. Semenova, PhD, ScD; Lyubov I. Kolesnikova, $\mathrm{PhD}, \mathrm{ScD}$, Member of the RAS; \\ Sergey I. Kolesnikov, $\mathrm{PhD}, \mathrm{ScD}$, Member of the RAS \\ Scientific Centre for Family Health and Human Reproduction Problems \\ Irkutsk, the Russian Federation
}

\begin{abstract}
Background: The aim of this study was to estimate the blood levels of interleukins (ILs) and tissue factor (TF) in rat blood when modeling acute mesenteric ischemia (AMI) in various stages and during reperfusion.

Methods and Results: The study was performed on white non-linear male rats, weighing $200 \pm 25 \mathrm{~g}$. AMI was simulated by ligating the cranial mesenteric artery at the mesentery root. Then, at certain time intervals, a relaparotomy was performed, blood was collected, and the animals were subjected to reperfusion at different time intervals.

During the experiment, the animals were divided into 3 groups depending on the time of ischemia ( 3 hours [n=10], 6 hours $[\mathrm{n}=10]$, and 8 hours [ $\mathrm{n}=10])$ followed by reperfusion after the indicated time of ischemia.

The level of ILs (IL-6, IL-8, and IL-10) and TF in rat blood serum was determined by EIA. We found that AMI in rats is accompanied by pronounced changes in the immune system and the development of a systemic inflammatory reaction syndrome, which is aggravated by reperfusion. At the same time, the restoration of blood flow after 3-hour ischemia was characterized by an increase in the concentration of IL-6, IL-8, and IL-10; after 6-hour ischemia - by an increase in the IL-6, IL-8 content and $\mathrm{TF}$, and stabilization of the IL-10 concentration. Reperfusion after 8-hour ischemia was accompanied by an increase in the IL-6 concentration, a decrease in the levels of IL-8, IL-10, and TF, which can lead to the progression of necrotic changes in the intestine.

Conclusion: The detected changes can serve as laboratory markers that characterize the course and stage of acute mesenteric ischemia. (International Journal of Biomedicine. 2020;10(4):416-420.)
\end{abstract}

Key Words: acute mesenteric ischemia $\bullet$ rats $\bullet$ immunity $\bullet$ lymphocytes

\section{Abbreviations}

AMI, acute mesenteric ischemia; EIA, enzyme immunoassay; IL, interleukin; TF, tissue factor.

\section{Introduction}

Despite the current progress in understanding the pathogenetic mechanisms of acute mesenteric ischemia (AMI), AMI remains a serious diagnostic problem, which contributes to maintaining a high level of mortality in patients. ${ }^{(1)}$ Early

*Corresponding author: Marina A. Darenskaya, PhD, ScD. Scientific Centre for Family Health and Human Reproduction Problems, Irkutsk, the Russian Federation. E-mail: marina darenskaya@inbox.ru diagnosis and rapid surgical intervention are necessary for adequate recovery of mesenteric blood flow, prevention of intestinal necrosis, and improvement of the clinical outcome of the disease.(2) Unsatisfactory results of this pathology's diagnostics are mainly due to the lack of a typical clinical picture, the low sensitivity of ultrasound and laparoscopic types of research, low availability of accurate diagnostic methods, and the lack of specific laboratory markers of the disease..$^{(3)}$

Cytokines play an important role in the development and course of various diseases, including those of the digestive organs. ${ }^{(4)}$ With the help of these specific proteins, a 
wide interaction is realized at the subcellular, cellular, organ, and system levels, forming a complex protective reaction aimed at neutralizing pathogens, destroying them, eliminating them from the body, and preserving the body's structural and functional integrity. ${ }^{(5)}$ In the body, cytokines interact closely with each other, forming a universal biological communication network that triggers and regulates a cascade of inflammatory, immune, and metabolic processes. ${ }^{(6)}$

An important factor involved in the formation of atherothrombosis is the tissue factor (TF), which is also closely related to the immune-inflammatory process, endothelial dysfunction, angiogenesis, and cell migration processes, all of which play a significant role not only in the development of cardiovascular diseases but also in the occurrence of a number of inflammatory and oncological processes. ${ }^{(7)}$ Clarification of AMI pathogenesis based on the analysis of immunological indicators will help to improve the quality of diagnostics and improve pathogenetic therapy, which will improve the results of care for patients with this pathology.

The aim of this study was to estimate the blood levels of interleukin and TF in rat blood when modeling $\mathrm{AMI}$ in various stages and during reperfusion.

\section{Materials and Methods}

The study was performed on white non-linear male rats, weighing $200 \pm 25 \mathrm{~g}$. The experiment was conducted on the basis of a certified vivarium of the Chita State Medical Academy. AMI was simulated by ligating the cranial mesenteric artery at the mesentery root. ${ }^{(8)}$ Termination of the blood flow through the artery was controlled visually: paling of the artery distal to the ligation site, desolation of the marginal vessels of the small intestine, and the cessation of their pulsation. After the operation, the animal was placed in a separate box, where it remained until the end of the experiment. Then, at certain time intervals, a relaparotomy was performed, blood was collected, and the animals were subjected to reperfusion at different time intervals. The resumption of blood flow through the artery was monitored visually: filling of the artery distal to the ligation site, filling of the marginal vessels of the small intestine, and the appearance of their pulsation. The wound was sutured; the animal was placed in a separate box. A day after reperfusion, under general anesthesia, a relaparotomy was performed, as well as intestinal viability assessment, blood sampling, and histological material sampling; then the animals were withdrawn from the experiment.

During the experiment, the animals were divided into 3 groups depending on the time of ischemia ( 3 hours [ $\mathrm{n}=10]$, 6 hours [n=10], and 8 hours $[\mathrm{n}=10]$ ) followed by reperfusion after the indicated time of ischemia.

The level of ILs and TF in rat blood serum was determined by EIA using test kits Cusabio Biotech Co., Ltd (China) for IL-6, Ray Biotech, Inc. (Germany) for IL-8 and IL-10, and Rat Tissue Factor (TF) ELISA (USA). All stages of the reaction were carried out on ST-3 shaker-incubators (Latvia) under thermostatically controlled conditions. Reaction tracking, calibration graphs, and concentration determination were performed on the ANTHOS 2010 vertical scanning photometer (Austria) using LabTech software.

The operations and all manipulations with animals were performed using general anesthesia, and euthanasia - by overdosing on drugs for anesthesia, taking into account the provisions regulated by ethical standards. Work on the animals was done in compliance with the principles of the Helsinki Declaration on the humane treatment of animals, stated in normative documents of the European community(86/609/ EU), Manual on Experimental (Preclinical) Study of New Pharmacological Substances, ${ }^{(9)}$ and "Good laboratory practice" (MHRF Order No. 708H dated 23.08.2010).

The study was approved by the Ethics Committee of the Chita State Medical Academy.

Statistical analysis was performed using the Statistica 6.1 software package (Stat-Soft Inc., USA). The normality of distribution of continuous variables was tested by the Kolmogorov-Smirnov test with the Lilliefors correction and Shapiro-Wilk test. For descriptive analysis, results are presented as mean \pm standard deviation (SD). The Wilcoxon criterion was used to compare the differences between the paired samples. A probability value of $P \leq 0.05$ was considered statistically significant.

\section{Results and Discussion}

When studying the dynamics of cytokines in the blood of experimental animals on the AMI model, the indicators during ischemia were compared with the initial data, and the parameters after reperfusion were compared with similar indicators during ischemia.

After 3 hours of ischemia, we found an increase in the serum content of IL- 6 and IL- 8 by 10.1 times $(P<0.01)$ and 4.1 times $(P<0.05)$, respectively, relative to the initial data (Fig.1).

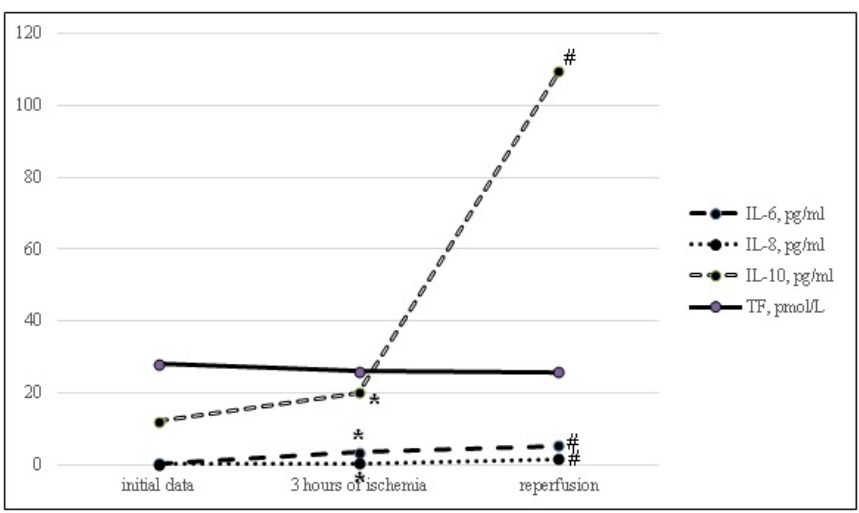

Fig. 1. Changes in the levels of ILs and TF after 3-hour ischemia and reperfusion (* - statistically significant differences with initial data, \# - statistically significant differences between 3-hour ischemia and reperfusion).

Recovery of mesenteric blood circulation (reperfusion), in comparison with data after 3-hour ischemia, caused an even greater increase in the IL- 6 concentration by 1.53 times $(P<0.05)$ and IL-8 by 3.47 times $(P<0.05)$. The level of 
IL-10 after 3 hours of ischemia increased by 1.67 relative to the initial level $(P<0.05)$, while reperfusion caused a sharp increase in the cytokine concentration - by 5.5 times, compared to data with ischemia $(P<0.01)$. No statistically significant results were obtained for the TF indicator $(P>0.05)$.

After 6 hours of ischemia, the serum concentration of IL-6 increased by 17.35 times $(P<0.01)$, in comparison with the initial data, and reperfusion contributed to the growth of this indicator by 1.45 times $(P<0.05)$ (Fig.2). A significant increase in IL-8 was observed after reperfusion - 7.32 times higher $(P<0.05)$ than the values of 6 -hour ischemia. The IL10 level increased by 1.58 times $(P<0.05)$ in relation to the initial data and did not change after reperfusion. The TF index decreased by 1.34 times $(P<0.05)$, compared to the initial value, and by 1.93 times $(P<0.05)$ after reperfusion.

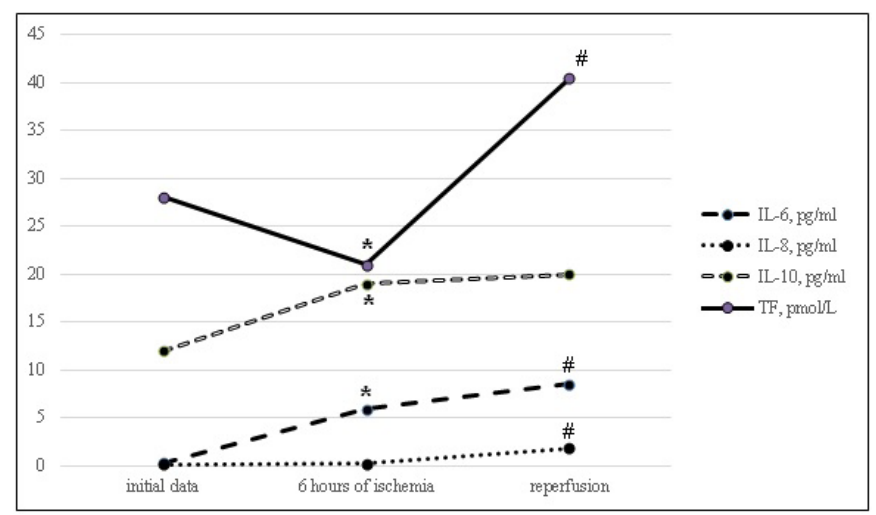

Fig. 2. Changes in the levels of ILs and TF after 6-hour ischemia and reperfusion (* - statistically significant differences with initial data, \# - statistically significant differences between 6-hour ischemia and reperfusion).

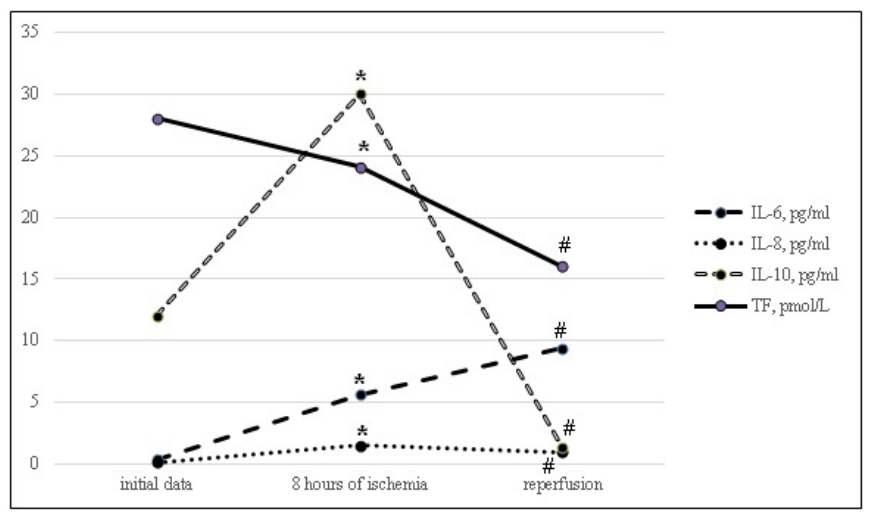

Fig. 3. Changes in the levels of ILs and TF after 8-hour ischemia and reperfusion (* - statistically significant differences with initial data, \# - statistically significant differences between 8-hour ischemia and reperfusion).

After 8 hours of ischemia, the serum concentration of IL-6 and IL-8 increased by 16.56 times $(P<0.01)$ and 12.33 times $(P<0.05)$, respectively, compared to the initial data
(Fig.3). At the same time, reperfusion contributed to an increase in IL- 6 by 1.67 times $(P<0.05)$ and a decrease 11 in IL- 8 by 1.51 times $(P<0.05)$. The content of IL-10 increased by 2.5 times $(P<0.05)$ from the baseline level and fell after reperfusion by 23.08 times $(P<0.05)$ relative to the data of 8 -hour ischemia. The TF index decreased by 1.16 times $(P<0.05)$ from the initial level and by 1.5 times $(P<0.05)$ after reperfusion.

By analogy with the processes occurring during the development of ischemia of other organs, it can be assumed that the very change in the intensity of blood flow in the ischemic tissue through the participation of mechanoreceptors can already serve as a signal to start the synthesis of proinflammatory cytokines. ${ }^{(5)}$ Developing hypoxia leads to a subsequent increase in the level of oxygen radicals, osmotic disorders, and damage to cell biomembranes. ${ }^{(10,11-14)}$ Thus, cytokines synthesized during the development of aseptic inflammation serve as endogenous mediators of the development of post-ischemic disorders in tissues. ${ }^{(15)} \mathrm{IL}-6$ is synthesized by many types of cells that trigger and regulate inflammation and immune response, and has an extremely versatile biological effect. ${ }^{(7)}$ The properties of IL-8 to cause cell migration and promote cell adhesion define it as an active participant in acute inflammatory response at the pathogen penetration sites. ${ }^{(5,6)}$ When studying the blood plasma of rats for the IL- 6 and IL-8 content, we noted their marked increase from the initial values to the eighth hour of intestinal ischemia. IL-10 is an anti-inflammatory cytokine produced by monocytes, macrophages, and activated CD4 cells. Attention is drawn to the ability of macrophages themselves to produce this cytokine, which is a strong inhibitor for them. ${ }^{(6)}$ IL-10 is known to promote humoral immune response and suppress the cellular immune response, as well as being a natural inhibitor of the nuclear factor, which plays a universal role in the development of an inflammatory cascade of various etiologies. ${ }^{(4,5)} \mathrm{We}$ observed an increase in this indicator during acute mesenteric ischemia, with maximum values after 8 hours of ischemia.

$\mathrm{TF}$ catalyzes the conversion of the inactive plasminogen to the active plasmin and is an important component of the fibrinolysis system. ${ }^{(6)}$ It is also one of the enzymes most often involved in the processes of destruction of the basement membrane, extracellular matrix, and cell invasion. The study of the TF content in the blood during the AMI simulation showed a decrease in its values during the entire period of ischemia, relative to the initial indicators, which can be explained by an increased expenditure of the factor in conditions of ischemic damage to intestinal tissues.

Next, we studied the effect of reperfusion after different periods of ischemia on the dynamics of IL-6, IL-8, IL-10, and TF indicators, depending on the time of ischemia. The recovery of mesenteric blood circulation after 3-hour ischemia, caused an increase in the levels of IL-6, IL-8, and IL-10, which indicated the predominance of anti-inflammatory cytokine potential in reperfusion at this time of ischemia. The reperfusion after 6-hour ischemia caused an increase in the levels of IL-6, IL-8, and TF. Thus, 
reperfusion at this time of ischemia was characterized by a predominance of proinflammatory cytokine potential. Restoring of the mesenteric circulation after 8-hour ischemia was characterized by an increase in the IL- 6 concentration and a decrease in the IL-8 concentration. It is noteworthy that the restoration of blood circulation at this time led to a sharp decrease in the IL-10 concentration, which can be explained by the severity of the pathological process. In addition, there was a decrease in the TF concentration, relative to the indicators before reperfusion, which is probably due to the expenditure of the latter in the conditions of ongoing necrotic changes in the intestine. It is also impossible to exclude the progression of mesenteric artery thrombosis, which can prevent the "leaching" of the factor into the general blood flow.

Treatment of acute mesenteric ischemiais primarily aimed at restoring blood circulation in the mesenteric bed. ${ }^{(16)}$ However, although the restoration of blood flow is essential in the mitigation of ischemic damage, reperfusion can initiate a whole series of inflammatory reactions and cause a local cellular response with the inclusion of molecular mechanisms. ${ }^{(17)}$ It was found that as a result of reperfusion, oxygen flow to damaged cells is restored, as a result of which the mitochondria begin to produce active oxygen metabolites, which lead to tissue damage. ${ }^{(18-21)}$ Cells die not at the height of ischemia, but after complete or partial restoration of blood circulation, as a result of increased oxidative stress reactions. ${ }^{(22)}$

Thus, we found that intestinal ischemia in rats for 8 hours is accompanied by pronounced changes in the immune system and the development of a systemic inflammatory reaction syndrome, which is aggravated by reperfusion. The detected changes can serve as laboratory markers that characterize the course and stage of acute mesenteric ischemia.

\section{Competing Interests} interests.

The authors declare that they have no competing

\section{References}

1. Al-Diery H, Phillips A, Evennett N, Pandanaboyana S, Gilham M, Windsor JA. The Pathogenesis of Nonocclusive Mesenteric Ischemia: Implications for Research and Clinical Practice. J Intensive Care Med. 2019 Oct;34(10):771-781. doi: 10.1177/0885066618788827.

2. van Dijk LJ, van Noord D, de Vries AC, Kolkman JJ, Geelkerken RH, Verhagen HJ, Moelker A, Bruno MJ. Clinical management of chronic mesenteric ischemia. United European Gastroenterol J. 2019 Mar;7(2):179-188. doi: $10.1177 / 2050640618817698$.

3. Gnanapandithan K, Feuerstadt P. Review Article: Mesenteric Ischemia. Curr Gastroenterol Rep. 2020 Mar 17;22(4):17. doi: 10.1007/s11894-020-0754-x.

4. Choghakhori R, Abbasnezhad A, Hasanvand A, Amani R. Inflammatory cytokines and oxidative stress biomarkers in irritable bowel syndrome: Association with digestive symptoms and quality of life. Cytokine. 2017 May;93:34-43. doi: 10.1016/j.cyto.2017.05.005.

5. Kany S, Vollrath JT, Relja B. Cytokines in Inflammatory Disease. Int J Mol Sci. 2019;20(23):6008. Published 2019 Nov 28. doi:10.3390/ijms20236008

6. Derikx JP, Schellekens DH, Acosta S. Serological markers for human intestinal ischemia: A systematic review. Best Pract Res Clin Gastroenterol. 2017 Feb;31(1):69-74. doi: 10.1016/j. bpg.2017.01.004.

7. Te Winkel JP, Drucker NA, Morocho BS, Shelley WC, Markel TA. Interleukin-6 Therapy Improves Intestinal Recovery Following Ischemia. J Surg Res. 2019 Jul;239:142148. doi: 10.1016/j.jss.2019.02.001.

8. Artamonova ZA, Namokonov EV, Tereshkov PP, Rusaeva NS. [Method for the diagnosis of intestinal necrosis in mesenteric ischemia in experiment]. Patent RU $2552338 \mathrm{C} 1$, 10.06.2015. Application №. 2014110453/15 of 03.18.2014. [In Russian].

9. Manual on Experimental (Preclinical) Study of New Pharmacological Substances. Ed. R. U. Khabriev. Moscow, 2005. [in Russian].

10. Darenskaya MA, Grebenkina LA, Sholokhov LF, Rashidova MA, Semenova NV, Kolesnikov S.I., et al. Lipid peroxidation activity in women with chronic viral hepatitis. Free Radical Biology \& Medicine. 2016;100(S):S192.

11. Kolesnikova LI, Kolesnikov SI, Darenskaya MA, Grebenkina LA, Nikitina OA, Lazareva LM, et al. Activity of LPO Processes in Women with Polycystic Ovarian Syndrome and Infertility. Bull Exp Biol Med. 2017;162(3):320-322. doi:10.1007/s10517-017-3605-5

12. Kolesnikova LI, Rychkova LV, Kolesnikova LR, Darenskaya MA, Natyaganova LV, Grebenkina LA, et al. Coupling of Lipoperoxidation Reactions with Changes in Arterial Blood Pressure in Hypertensive ISIAH Rats under Conditions of Chronic Stress. Bull Exp Biol Med. 2018;164(6):712-715. doi:10.1007/s10517-018-4064-3

13. Kolesnikova LI, Kolesnikov SI, Romanova ED, Chkhenkeli VA, Darenskaya MA, Grebenkina LA, et al. Effect of Preparation Based on Trametes Pubescens Xylotroph Fungi on Lipid Peroxidation in the Blood of Experimental Animals under Conditions of Dark Stress. Bull Exp Biol Med. 2017;162(6):762-764. doi:10.1007/s10517017-3707-0

14. Kolesnikova LI, Kolesnikova LR, Darenskaya MA, Natyaganova LV, Grebenkina LA, Korytov LI, et al. Evaluation of Lipid Peroxidation-Antioxidant Defense System Depending on the Stage of Stress Reaction in Hypertensive ISIAH Rats. Bull Exp Biol Med. 2019;166(5):610-612. doi:10.1007/ s10517-019-04402-6

15. Toldo S, Mauro AG, Cutter Z, Abbate A. Inflammasome, pyroptosis, and cytokines in myocardial ischemia-reperfusion injury. Am J Physiol Heart Circ Physiol. 2018 Dec 1;315(6):H1553-H1568. doi: 10.1152/ajpheart.00158.2018.

16. Khripun AI, Shurygin SN, Mironkov AB, Izvekov AA, Guseva TV, Pryamikov AD. [Microcirculation in the small and large intestine with thrombosis and thromboembolism of the mesenteric arteries]. Khirurgiya. Zhurnal imeni N.I. Pirogova. 2011;9:27-32. [In Russian]

17. Bertoni S, Ballaben V, Barocell E, Tognolini M. Mesenteric ischemia-reperfusion: an overview of preclinical drug strategies. Drug Discov Today. 2018;23(7):1416-1425. doi:10.1016/j.drudis.2018.05.034

18. Kolesnikova LR, Darenskaya MA, Rychkova LV, Pogodina AV, Grebenkina LA, Kolesnikov SI, et al. Oxidative 
stress parameters and state of regional periodontal blood flow in adolescents with arterial hypertension and periodontal diseases. International Journal of Biomedicine. 2018;8(4):301305. doi:10.21103/Article8(4)_OA6

19. Darenskaya MA, Kolesnikov SI, Rychkova LV, Grebenkina LA, Kolesnikova LI. Oxidative stress and antioxidant defense parameters in different diseases: ethnic aspects. Free Radical Biology \& Medicine. 2018;120(S1):60.

20. Bairova TA, Kolesnikov SI, Kolesnikova LI, Pervushina OA, Darenskaya M.A, Grebenkina LA. Lipid peroxidation and mitochondrial superoxide dismutase-2 gene in adolescents with essential hypertension. Bull Exp
Biol Med. 2014;158(2):181-184. doi:10.1007/s10517-0142717-4

21. Kolesnikova LI, Darenskaya MA, Kolesnikov SI. [Free radical oxidation: pathophysiologist's view]. Bulletin of Siberian medicine. 2017;16(4):16-29. [Article in Russian]. 22. Poles MZ, Bódi N, Bagyánszki M, Fekete É, Mészáros AT, Varga G, Szücs S, Nászai A, Kiss L, Kozlov AV, Boros M, Kaszaki J. Reduction of nitrosative stress by methane: Neuroprotection through xanthine oxidoreductase inhibition in a rat model of mesenteric ischemia-reperfusion. Free Radic Biol Med. 2018 May 20;120:160-169. doi: 10.1016/j. freeradbiomed.2018.03.024. 